\title{
CLASSIFICATION OF HOLOMORPHIC PFAFF SYSTEMS ON HOPF MANIFOLDS
}

\author{
MAURÍCIO CORRÊA*, ANTONIO M. FERREIRA, AND MISHA VERBITSKY
}

\begin{abstract}
We classify holomorphic Pfaff systems (possibly non locally decomposable) on certain Hopf manifolds. As consequence, we prove some integrability results. We also prove that any holomorphic distribution on a general (non-resonance) Hopf manifold is integrable.
\end{abstract}

\section{INTRODUCTION}

Let $W=\mathbb{C}^{n}-\{0\}, n \geq 2$ and $f\left(z_{1}, z_{2}, \ldots, z_{n}\right)=\left(\mu_{1} z_{1}, \mu_{2} z_{2}, \ldots, \mu_{n} z_{n}\right)$ be a diagonal contraction in $\mathbb{C}^{n}$, where $0<\left|\mu_{i}\right|<1$ for all $1 \leq i \leq n$. The quotient space $X=W /\langle f\rangle$ is a compact complex manifold of dimension $n$ called a diagonal Hopf manifold. When $\mu_{1}=\cdots=\mu_{n}$ we say that $X$ is a classical Hopf manifold. Classical Hopf manifolds were first studied by Heinz Hopf [Ho] in 1948. Hopf showed that $X$ is diffeomorphic to the product of odd spheres $S^{1} \times S^{2 n-1}$ and has a complex structure which is not-Kähler. K. Kodaira [Ko] classified Hopf surfaces. The geometry of Hopf manifolds have been studied by several authors, see for instance, Dabrowski [Da], Haefliger [Ha], Kato [Kat], Ise [I], Wehler[W] etc.

A holomorphic singular Pfaff system $\mathcal{F}$, of codimension $k$, on $X$ is a non-trivial section $\omega \in \mathrm{H}^{0}\left(X, \Omega_{X}^{k} \otimes \mathcal{L}\right)$, where $\mathcal{L}$ is a holomorphic line bundle on $X$. In this work we will study the geometry and classification of holomorphic Pfaff systems on Hopf manifolds.

We will consider the following types of Hopf manifolds: 1

Definition 1.1. We say that

(1) $X$ is classical if $\mu=\mu_{1}=\cdots=\mu_{n}$.

(2) $X$ is no-resonance if $0<\left|\mu_{1}\right| \leq\left|\mu_{2}\right| \leq \ldots \leq\left|\mu_{n}\right|<1$ and there is no non-trivial relation among the $\mu_{i}$ 's in this way

$$
\prod_{i \in A} \mu_{i}^{r_{i}}=\prod_{j \in B} \mu_{j}^{r_{j}}, \quad r_{i}, r_{j} \in \mathbb{N}, \quad A \cap B=\emptyset, \quad A \cup B=\{1,2, \ldots, n\} .
$$

(3) $X$ is weak no-resonance if $\mu_{1}=\mu_{2}=\cdots=\mu_{r}$, where $2 \leq r \leq n-1$ and there is no non-trivial relation among the $\mu_{i}$ 's in this way

$$
\prod_{i \in A} \mu_{i}^{r_{i}}=\prod_{j \in B} \mu_{j}^{r_{j}}, \quad r_{i}, r_{j} \in \mathbb{N}, \quad A \cap B=\emptyset, \quad A \cup B=\{1, r+1, \ldots, n\} .
$$

2010 Mathematics Subject Classification. Primary 32S65, 37F75, 32M25.

Key words and phrases. Pfaff systems, Holomorphic distributions and foliations, Hopf manifolds.

${ }^{*}$ Corresponding author.

${ }^{1}$ In the work $\mathrm{CFF}$ the authors have used other terminology. 
Remark 1.2. Note that a general Hopf manifold is always no-resonance. Indeed, each of the algebraic conditions $\prod_{i \in A} \mu_{i}^{r_{i}}=\prod_{j \in B} \mu_{j}^{r_{j}}$ gives a closed algebraic subvariety of positive codimension in the space of all operators $A$. Therefore, the set of no-resonance Hopf manifold is obtained as a complement to a countable union of such subvarieties.

D. Mall in Ma1 used the Kodaira's classification of Hopf surfaces to obtain a complete description of regular holomorphic foliations on Hopf surfaces. E. Ghys in [G], studied regular holomorphic foliations on homogeneous spaces, and as a consequence obtained the classification of codimension one foliation on classical Hopf manifolds.

In CFF] M. Corrêa, A. Fernández-Pérez, and A. M. Ferreira classified regular holomorphic foliations of dimension and codimension one on certain Hopf manifolds. As a consequence of the classification the authors proved that any regular codimension one distribution on a weak no-resonance or no-resonance Hopf manifold is integrable and admits a holomorphic first integral.

M. Ise proved in [I] that if $X$ is a classical Hopf manifold, then a line bundle $L$ on $X$ is the quotient of $W \times \mathbb{C}$ by the operation of a representation of the fundamental group of $X \varrho_{L}: \pi_{1}(X) \simeq \mathbb{Z} \longrightarrow G L(1, \mathbb{C})=\mathbb{C}^{*}$ in the following way

$$
\begin{array}{ccc}
W \times \mathbb{C} & \longrightarrow & W \times \mathbb{C} \\
(z, v) & \longmapsto & \left(f(z), \varrho_{L}(1) v\right)
\end{array}
$$

In Ma2 M. Mall has generalized this fact for arbitrary Hopf manifolds. We will write $L=L_{b}$ for the line bundle induced by the representation $\varrho_{L}(\gamma)$ with $b=\varrho_{L}(1)$.

In this paper we will show the following:

Theorem 1.3. Let $X$ be a Hopf manifold and let $\mathcal{F}$ be a singular holomorphic Pfaff system on $X$, of codimension $k$, given by a nonzero twisted differential $k$ form $\omega \in H^{0}\left(X, \Omega_{X}^{k} \otimes L_{b}\right)$ with coefficients in the line bundle $L_{b}$. Consider the natural projection $\pi: W \rightarrow X$. Then the following holds:

(i) If $X$ is classical, then $b=\mu^{m}$ with $m \in \mathbb{N}$ and $m \geq k$. Furthermore $\pi^{*} \omega$ is a homogeneous polynomial $k$-form of degree $m-k$.

(ii) If $X$ is no-resonance, then $b=\mu_{1}^{m_{1}} \ldots \mu_{n}^{m_{n}}$ such that $m_{j} \in\{0,1\}$ for all $j=1, \ldots, n$. Furthermore, $\pi^{*} \omega$ is a monomial $k$-form of the type

$$
\sum_{i_{1}<\cdots<i_{k}} g_{i_{1} \ldots i_{k}} d z_{i_{1}} \wedge \cdots \wedge d z_{i_{k}}
$$

with

$$
g_{i_{1} \ldots i_{k}}\left(z_{1}, \ldots, z_{n}\right)=c_{m}^{i_{1} \ldots i_{k}} z_{j_{1}}^{m_{j_{1}}} z_{j_{2}}^{m_{j_{2}}} \ldots z_{j_{n-k}}^{m_{j_{n-k}}}
$$

where $\left\{j_{1}, j_{2}, \ldots, j_{n-k}\right\}=\{1, \ldots, n\} \backslash\left\{i_{1}, \ldots, i_{k}\right\}$.

(iii) If $X$ is weak no-resonance, then $b=\mu^{m} \mu_{r+1}^{m_{r+1}} \ldots \mu_{n}^{m_{n}}$ with $m \in \mathbb{N}$, and $m_{j} \in\{0,1\}$ for all $j=r+1, \ldots, n$. Furthermore, $\pi^{*} \omega$ is a polinomial $k$-form of the type

$$
\pi^{*} \omega=\sum_{i_{1}<\cdots<i_{k}} g_{i_{1} \ldots i_{k}} d z_{i_{1}} \wedge \cdots \wedge d z_{i_{k}},
$$


where

$$
\begin{aligned}
& g_{i_{1} \ldots i_{k}}=z_{j_{1}}^{m_{j_{1}}} \ldots z_{j_{n-r-k+s}}^{m_{j_{n-k-k}}} \cdot \sum_{\alpha_{1}+\cdots+\alpha_{r}=m-s} c_{\alpha}^{i_{1} \ldots i_{k}} z_{1}^{\alpha_{1}} \ldots z_{r}^{\alpha_{r}} \\
& \text { such that }\left\{j_{1}, \ldots, j_{n-r-k+s}\right\}=\{r+1, \ldots, n\} \backslash\left\{i_{s+1}, \ldots, i_{k}\right\} \text { and } s \in \\
& \{0, \ldots, k\} \text { is defined in such a way that } i_{1}, \ldots, i_{s} \leq r \text { and } r+1 \leq i_{s+1}, \ldots, i_{k} .
\end{aligned}
$$

The part $i$ ) of the Theorem 1.3 generalizes the result in $\mathrm{G}$ in the codimension one case and [CFF in the one-dimensional case.

We obtain the following consequence for regular Pfaff system on no-resonance Hopf manifolds.

Corollary 1.4. Any regular holomorphic Pfaff system of codimension $k$ on a noresonance Hopf manifold is integrable and has a compact leaf.

For singular holomorphic distributions we show the following integrability result.

Theorem 1.5. Let $\mathcal{F}$ be a singular holomorphic distribution on a no-resonance Hopf manifold $X$. Then $\mathcal{F}$ is integrable.

Acknowledgments. Maurício Corrêa is supported by the CNPq grant numbers 202374/2018-1, 302075/2015-1, 400821/2016-8. Misha Verbitsky is supported by Russian Academic Excellence Project '5-100", CNPq - Process 313608/2017-2, and FAPERJ E-26/202.912/2018. We would like to thank the referee for precious comments which improved the presentation of the paper greatly.

\section{Pfaff systems, Distributions and Holomorphic foliations}

Let $X$ be an $n$-dimensional complex manifold.

Definition 2.1. A holomorphic singular Pfaff system $\mathcal{F}$, of codimension $k$, on $X$ is a non-trivial section $\omega \in \mathrm{H}^{0}\left(X, \Omega_{X}^{k} \otimes \mathcal{L}\right)$, where $\mathcal{L}$ is a holomorphic line bundle on $X$. The singular set of $\omega$ is defined by

$$
\operatorname{Sing}(\mathcal{F}):=\operatorname{Sing}(\omega)=\{z \in X ; \omega(z)=0\} .
$$

We suppose that $\operatorname{cod}(\operatorname{Sing}(\omega)) \geq 2$. We say that $\omega$ is regular (or non-singular ) if $\operatorname{Sing}(\omega)=\emptyset$.

Remark 2.2. Sometimes a Pfaff system is defined as a saturated rank 1 subsheaf in $\Omega_{X}^{k}$. This definition is not equivalent to the one given above because of ambiguity the choice of the line bundle $\mathcal{L}$. However, for most practical purposes there notions are the same.

Given a Pfaff system $\mathcal{F}$ on $X$ induced by $\omega \in \mathrm{H}^{0}\left(X, \Omega_{X}^{k} \otimes \mathcal{L}\right)$, then $\omega$ is determined by the following:

(i) a open covering $\left\{U_{\alpha}\right\}_{\alpha \in \Lambda}$ of $X$;

(ii) holomorphic $k$-forms $\omega_{\alpha} \in \Omega_{U_{\alpha}}^{k}$ satisfying $\omega_{\alpha}=h_{\alpha \beta} \omega_{\beta}$ on $U_{\alpha} \cap U_{\beta} \neq \emptyset$,

where $h_{\alpha \beta} \in \mathcal{O}\left(U_{\alpha} \cap U_{\beta}\right)^{*}$ determines the cocycle representing $\mathcal{L}$. Therefore, the singular set of $\mathcal{F}$ is $\cup_{\alpha \in \Lambda}\left\{\omega_{\alpha}=0\right\}$. For more details about Pfaff systems see EK, CJV, CMS].

A distribution on $X$ of codimension $k$ is a nonzero coherent subsheaf $\mathcal{G} \subset \Omega_{X}^{1}$ of rank $k$ such that $\mathcal{G}$ is saturated in $\Omega_{X}^{1}$ (i.e., $\Omega_{X}^{1} / \mathcal{G}$ is torsion free). 
The $k$-th wedge product of the inclusion $\mathcal{G} \subset \Omega_{X}^{1}$ gives rise to a twisted holomorphic differential $k$-form with coefficients in the line bundle $\operatorname{det}(\mathcal{G})$. That is, a distribution of codimension $k$ induces a Pfaff system

$$
\omega \in \mathrm{H}^{0}\left(X, \Omega_{X}^{k} \otimes \operatorname{det}(\mathcal{G})\right)
$$

of codimension $k$ which is locally decomposable outside its singular set. That is, for each point $p \in X \backslash \operatorname{Sing}(\omega)$ there exists a neighbourhood $U$ and holomorphics 1 -forms $\omega_{1}, \ldots, \omega_{k} \in H^{0}\left(U, \Omega_{U}^{1}\right)$ such that

$$
\left.\omega\right|_{U}=\omega_{1} \wedge \cdots \wedge \omega_{k}
$$

A singular holomorphic distribution $\mathcal{F}$ is a foliation if it is integrable, i.e, if

$$
d \omega_{i} \wedge \omega_{1} \wedge \cdots \wedge \omega_{k}=0
$$

for all $i=1, \ldots, k$. The tangent sheaf of a distribution $\mathcal{F}$ induced by a twisted form $\omega$ is

$$
T \mathcal{F}=\left\{v \in T X ; i_{v} \omega=0\right\} .
$$

The rank of $T \mathcal{F}$ is $(n-k)$ and by integrability condition we have that $[T \mathcal{F}, T \mathcal{F}] \subset$ $T \mathcal{F}$.

There is the following exact sequence of sheaves

$$
0 \rightarrow T \mathcal{F} \rightarrow T X \rightarrow N_{\mathcal{F}} \rightarrow 0
$$

where $N_{\mathcal{F}}$ is a torsion free sheaf of rank $k$. Conversely, given a nonzero coherent subsheaf $T \mathcal{F} \subset T X$ of rank $k$ such that $T X / T \mathcal{F}=N_{\mathcal{F}}$ is torsion free defines a distribution $\left(N_{\mathcal{F}}\right)^{*} \subset \Omega_{X}^{1}$.

We remark that by construction the tangent bundle of a Hopf manifold $X$ is given by

$$
T X=\bigoplus_{i=1}^{n} L_{\mu_{i}},
$$

where $L_{\mu_{i}}$ is the tangent bundle of the foliation induced by the canonical vector field $\frac{\partial}{\partial z_{i}}$.

\section{Cohomology of Line Bundles on Hopf Manifolds}

Let $W=\mathbb{C}^{n}-\{0\}, n \geq 2$ and $f\left(z_{1}, z_{2}, \ldots, z_{n}\right)=\left(\mu_{1} z_{1}, \mu_{2} z_{2}, \ldots, \mu_{n} z_{n}\right)$ be a diagonal contraction in $\mathbb{C}^{n}$, where $0<\left|\mu_{i}\right|<1$ for all $1 \leq i \leq n$. Consider the associated Hopf manifold $X=W /\langle f\rangle$.

Let $\Omega_{X}^{p}$ be the sheaf of germs of holomorphic $p$-forms on a Hopf manifold $X$. Denote by $\Omega_{X}^{p}\left(L_{b}\right):=\Omega_{X}^{p} \otimes L_{b}$, where $L_{b}$ is a line bundle determined by a character $b$ as in (1), and by $\pi: W \rightarrow X$ the natural projection on $X$.

We will adopt the notation in Ma2. Consider an open covering $A=\left\{U_{i}\right\}$ of $X$ such that $U_{i}$ are open and contractible Stein subsets of $X$ and $\tilde{U}_{i}:=\pi^{-1}\left(U_{i}\right)$ is a disjoint union of Stein open sets $\left\{U_{i j}^{\prime}\right\}$ of $W$, each of them isomorphic to $U_{i}$. We have that

$$
\tilde{U}_{i}=\bigcup_{r \in \mathbb{Z}} f^{r}\left(U_{i 0}\right) ; \quad \tilde{A}=\left\{\tilde{U}_{i}\right\} .
$$

Consider $\varphi \in \Gamma\left(U_{i}, \Omega_{X}^{p}\left(L_{b}\right)\right)$. Then

$$
\tilde{\varphi}=\pi^{*}(\varphi) \in \Gamma\left(\tilde{U}_{i}, \pi^{*}\left(\Omega_{X}^{p}\left(L_{b}\right)\right)\right) \cong \Gamma\left(\tilde{U}_{i}, \Omega_{W}^{p}\right) .
$$


Denote the map $p_{0}=b \cdot i d-f^{*}: H^{0}\left(W, \Omega_{W}^{p}\right) \rightarrow H^{0}\left(W, \Omega_{W}^{p}\right)$. We have an exact sequence of Cěch complexes

$$
0 \longrightarrow \mathcal{C} \cdot\left(A, \Omega_{X}^{p}\left(L_{b}\right)\right) \stackrel{\pi^{*}}{\longrightarrow} \mathcal{C} \cdot\left(\tilde{A}, \Omega_{W}^{p}\right) \stackrel{p_{0}}{\longrightarrow} \mathcal{C} \cdot\left(\tilde{A}, \Omega_{W}^{p}\right) \longrightarrow 0
$$

From (2) we derive the long exact sequence of cohomology

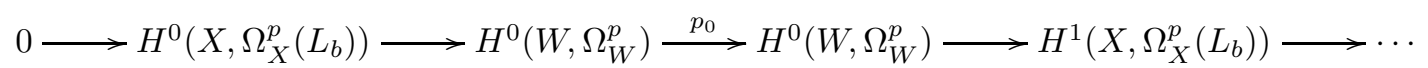

D. Mall proved in Ma2] the following result:

Theorem 3.1 (Mall Ma2]). If $X$ is a Hopf manifold, of dimension $n \geq 3$, and $L_{b}$ is a line bundle on $X$, then

$$
\operatorname{dim} H^{0}\left(X, \Omega_{X}^{p}\left(L_{b}\right)\right)=\operatorname{dim} \operatorname{Ker}\left(p_{0}\right) .
$$

In order to prove Theorem 1.3 we first prove the following lemma.

Lemma 3.2. Let $X$ be a classical, no-resonance or weak no-resonance Hopf manifold of dimension $n \geq 3$, and let $L_{b}$ be a line bundle on $X$, with $b \in \mathbb{C}^{*}$. The following holds:

(i) If $X$ is classical, then $\operatorname{dim} H^{0}\left(X, \Omega_{X}^{k} \otimes L_{b}\right)>0$ if and only if $b=\mu^{m}$, where $m \in \mathbb{N}, m \geq k$.

(ii) If $X$ is no-resonance, then $\operatorname{dim} H^{0}\left(X, \Omega_{X}^{k} \otimes L_{b}\right)>0$ if and only if

$$
b=\mu_{1}^{m_{1}} \mu_{2}^{m_{2}} \ldots \mu_{n}^{m_{n}}
$$

where $m_{i} \in \mathbb{N}$ and there exist $j_{1}, \ldots, j_{k} \in\{1, \ldots, n\}$, such that $m_{j_{1}}, \ldots, m_{j_{k}} \geq 1$.

(iii) If $X$ is weak no-resonance, then $\operatorname{dim} H^{0}\left(X, \Omega_{X}^{k} \otimes L_{b}\right)>0$ if and only if

$$
b=\mu_{1}^{m_{1}} \mu_{2}^{m_{2}} \ldots \mu_{n}^{m_{n}}
$$

with $m_{j} \in \mathbb{N}$ for all $j=1, \ldots, n, m_{1}+m_{2}+\cdots+m_{r}=t$, and there exist $i_{1}, \ldots, i_{k-t} \geq r+1$ such that $m_{i_{1}} \geq 1, \ldots, m_{i_{k-t}} \geq 1$.

Proof. From Theorem 3.1 we have that $\operatorname{dim} H^{0}\left(X, \Omega_{X}^{k} \otimes L_{b}\right)=\operatorname{dim}\left(k e r p_{0}\right)$, where the map

$$
p_{0}: H^{0}\left(W, \Omega_{W}^{k}\right) \longrightarrow H^{0}\left(W, \Omega_{W}^{k}\right),
$$

is given by $p_{0}=b \cdot i d-f^{*}$. Let $\omega \in H^{0}\left(W, \Omega_{W}^{k}\right)$ be. We write

$$
\omega=\sum_{i_{1}<\cdots<i_{k}} g_{i_{1} \ldots i_{k}} d z_{i_{1}} \wedge \cdots \wedge d z_{i_{k}} .
$$

It follows from Hartog's extension theorem that each $g_{i_{1} \ldots i_{k}}$ can be represented by its Taylor series

$$
g_{i_{1} \ldots i_{k}}\left(z_{1}, z_{2}, \ldots, z_{n}\right)=\sum_{\alpha \in \mathbb{N}^{n}} c_{\alpha}^{i_{1} \ldots i_{k}} z_{1}^{\alpha_{1}} z_{2}^{\alpha_{2}} \ldots z_{n}^{\alpha_{n}} \text {, for all } i=1, \ldots, n .
$$

Hence

$$
p_{0}(\omega)=\sum_{i_{1}<\cdots<i_{k}} \sum_{\alpha \in \mathbb{N}^{n}} c_{\alpha}^{i_{1} \ldots i_{k}}\left(b-\mu_{1}^{\alpha_{1}} \ldots \mu_{n}^{\alpha_{n}} \mu_{i_{1}} \ldots \mu_{i_{k}}\right) z_{1}^{\alpha_{1}} \ldots z_{n}^{\alpha_{n}} d z_{i_{1}} \wedge \cdots \wedge d z_{i_{k}} .
$$


In the classical case, $\mu_{1}=\cdots=\mu_{n}=\mu$ and thus $\operatorname{dim}\left(\operatorname{ker}\left(p_{0}\right)\right)>0$ if, and only if, $b=\mu^{m}$, for some $m \in \mathbb{N}, m \geq k$.

In the no-resonance case, since $\mu_{i}^{\prime} s$ have no relations, it follows from (3) that $\operatorname{dim}\left(\operatorname{ker}\left(p_{0}\right)\right)>0$ if, and only if, $b=\mu_{1}^{m_{1}} \mu_{2}^{m_{2}} \ldots \mu_{n}^{m_{n}}$ where $m_{j} \in \mathbb{N}$, and there exist $j_{1}, \ldots, j_{k} \in\{1, \ldots, n\}$, such that $m_{j_{1}}, \ldots, m_{j_{k}} \geq 1$.

Finally, for the weak no-resonance case, we have $\mu_{1}=\cdots=\mu_{r}=\mu$. Since $\mu, \mu_{r+1}, \ldots, \mu_{n}$ have no relations, we have $\operatorname{dim}\left(\operatorname{ker}\left(p_{0}\right)\right)>0$ if, and only if, $b=$ $\mu^{m} \mu_{r+1}^{m_{r+1}} \ldots \mu_{n}^{m_{n}}$ such that $m_{j} \in \mathbb{N}$ for all $j=1, \ldots, n, m_{1}+m_{2}+\cdots+m_{r}=t$, and there exist $i_{1}, \ldots, i_{k-t} \geq r+1$ such that $m_{i_{1}} \geq 1, \ldots, m_{i_{k-t}} \geq 1$.

\subsection{Proof of Theorem $\mathbf{1 . 3}$.}

Proof. First of all, we may assume that $k \leq n-2$, since the case when $k=n-1$ was done in $\mathrm{CFF}$. Consider the natural projection $\pi: W \rightarrow X$. By construction, we have that a holomorphic section $\omega \in H^{0}\left(X, \Omega_{X}^{k} \otimes L_{b}\right)$ corresponds to a non-trivial section

$$
\pi^{*} \omega=\left(g_{i_{1} \ldots i_{k}}\right)_{i_{1}<\cdots<i_{k}} \in H^{0}\left(W, \mathcal{O}_{W}^{\left(\begin{array}{c}
n \\
k
\end{array}\right)}\right),
$$

such that $g_{i_{1} \ldots i_{k}} \in \mathcal{O}_{W}$ satisfies

$$
g_{i_{1} \ldots i_{k}}\left(\mu_{1} z_{1}, \ldots, \mu_{n} z_{n}\right)=\mu_{i_{1}}^{-1} \ldots \mu_{i_{k}}^{-1} b g_{i_{1} \ldots i_{k}}\left(z_{1}, \ldots, z_{n}\right),
$$

for all $i_{1}<\cdots<i_{k}$. By Hartog's extension theorem, $\pi^{*} \omega$ can be represented on $\mathbb{C}^{n}$ by its Taylor series

$$
g_{i_{1} \ldots i_{k}}\left(z_{1}, \ldots, z_{n}\right)=\sum_{\alpha \in \mathbb{N}^{n}} c_{\alpha}^{i_{1} \ldots i_{k}} z_{1}^{\alpha_{1}} \ldots z_{n}^{\alpha_{n}}, \text { where } \alpha=\left(\alpha_{1}, \alpha_{2}, \ldots, \alpha_{n}\right) \in \mathbb{N}^{n} .
$$

Then

$$
c_{\alpha}^{i_{1} \ldots i_{k}} \mu_{1}^{\alpha_{1}} \mu_{2}^{\alpha_{2}} \ldots \mu_{n}^{\alpha_{n}}=c_{\alpha}^{i_{1} \ldots i_{k}} \mu_{i_{1}}^{-1} \ldots \mu_{i_{k}}^{-1} b
$$

where $\alpha=\left(\alpha_{1}, \alpha_{2}, \ldots, \alpha_{n}\right) \in \mathbb{N}^{n}$. Therefore, for all $c_{\alpha}^{i_{1} \ldots i_{k}} \neq 0$ we get that

$$
\mu_{1}^{\alpha_{1}} \mu_{2}^{\alpha_{2}} \ldots \mu_{n}^{\alpha_{n}}=\mu_{i_{1}}^{-1} \ldots \mu_{i_{k}}^{-1} b
$$

Classical case. In this case we have that $\mu_{1}=\cdots=\mu_{n}=\mu$. Lemma 3.2 item $(i)$ implies that $b=\mu^{m}$ for some $m \geq k$. Therefore, from (4) we have

$$
\mu^{|\alpha|}=\mu^{-k} \mu^{m}, \text { where }|\alpha|=\alpha_{1}+\cdots+\alpha_{n} .
$$

This implies that $|\alpha|=m-k$ for all $\alpha=\left(\alpha_{1}, \alpha_{2}, \ldots, \alpha_{n}\right) \in \mathbb{N}^{n}$ and $i_{1}<\cdots<i_{k}$ such that $c_{\alpha}^{i_{1} \ldots i_{k}} \neq 0$. It follows that each $g_{i_{1} \ldots i_{k}}$ is a homogeneous polynomial of degree $m-k$.

No-resonance case. If $X$ is no-resonance, then by Lemma 3.2 item $(i i)$ we have

$$
b=\mu_{1}^{m_{1}} \mu_{2}^{m_{2}} \ldots \mu_{n}^{m_{n}}
$$

where $m_{i} \in \mathbb{N}$ and there exist $l_{1}, \ldots, l_{k} \in\{1, \ldots, n\}$ such that $m_{l_{1}}, \ldots, m_{l_{k}} \geq 1$. So from (4) we get

$$
\mu_{1}^{\alpha_{1}} \mu_{2}^{\alpha_{2}} \ldots \mu_{n}^{\alpha_{n}}=\mu_{i_{1}}^{-1} \ldots \mu_{i_{k}}^{-1} \mu_{1}^{m_{1}} \mu_{2}^{m_{2}} \ldots \mu_{n}^{m_{n}}
$$


for all $\alpha=\left(\alpha_{1}, \alpha_{2}, \ldots, \alpha_{n}\right) \in \mathbb{N}^{n}$ and $i_{1}<\cdots<i_{k}$ such that $c_{\alpha}^{i_{1} \ldots i_{k}} \neq 0$. Since there is not non-trivial relation between the $\mu_{i}$ 's, we must have that $\alpha=\tilde{m}=$ : $\left(m_{1}, \ldots, m_{i_{1}}-1, \ldots, m_{i_{k}}-1 \ldots, m_{n}\right)$. Thus

$$
g_{i_{1} \ldots i_{k}}\left(z_{1}, \ldots, z_{n}\right)=c_{\tilde{m}}^{i_{1} \ldots i_{k}} z_{1}^{m_{1}} \ldots z_{i_{1}}^{m_{i_{1}}-1} \ldots z_{i_{k}}^{m_{i_{k}}-1} \ldots z_{n}^{m_{n}}
$$

Therefore the $k$-form $\pi^{*} \omega$ is monomial.

We must have that $m_{i} \in\{0,1\}$, for all $i \in\{1, \cdots, n\}$, otherwise, we would have that $\left\{z_{i}=0\right\} \subset \operatorname{Sing}\left(\pi^{*} \omega\right)$ for some $i$. A contradiction, since the Pfaff system has singular set with codimension $\geq 2$. Then, if the coefficient $g_{i_{1} \ldots i_{k}} \not \equiv 0$, we have from (5) that

$$
g_{i_{1} \ldots i_{k}}\left(z_{1}, \ldots, z_{n}\right)=c_{\tilde{m}}^{i_{1} \ldots i_{k}} z_{j_{1}}^{m_{j_{1}}} z_{j_{2}}^{m_{j_{2}}} \ldots z_{j_{n-k}}^{m_{j_{n-k}}}
$$

where $\left\{j_{1}, j_{2}, \ldots, j_{n-k}\right\}=\{1, \ldots, n\} \backslash\left\{i_{1}, \ldots, i_{k}\right\}$, and $m_{i_{1}}=\cdots=m_{i_{k}}=1$.

Now we will determine the regular $k$-forms.

3.1.1. Proof of Corollary 1.4. If $\pi^{*} \omega=\left(g_{i_{1} \ldots i_{k}}\right)_{i_{1}<\cdots<i_{k}}$ is regular, then there exist a coefficient $g_{h_{1} \cdots h_{k}}$, for some $\left(h_{1}, \ldots, h_{k}\right)$, such that it is not zero along $V_{1}=$ $\cap_{i=2}^{n}\left\{z_{i}=0\right\}$. We will consider the following subcases:

(1) suppose that $h_{1}=1$ : from equation (6) we have

$$
g_{h_{1} \ldots h_{k}}\left(z_{1}, \ldots, z_{n}\right)=c_{\tilde{m}}^{h_{1} \ldots h_{k}} z_{j_{1}}^{m_{j_{1}}} z_{j_{2}}^{m_{j_{2}}} \ldots z_{j_{n-k}}^{m_{j_{n-k}}}
$$

with $2 \leq j_{1} \leq \cdots \leq j_{n-k}$ and $m_{h_{1}}=\cdots=m_{h_{k}}=1$. Since $g_{h_{1} \cdots h_{k}}$ is not zero along $V_{1}$, we must have that $m_{j_{1}}=m_{j_{2}}=\cdots m_{j_{n-k}}=0$. Now, as we know all $m_{i}^{\prime} s$, from equation 6, we have that the only coefficient of the $k$-form $\pi^{*} \omega$ which is not identically zero is $g_{h_{1} \ldots h_{k}}=c_{\tilde{m}}^{h_{1} \ldots h_{k}}=$ constant. Therefore

$$
\pi^{*} \omega=c_{\tilde{m}}^{h_{1} \ldots h_{k}} d z_{h_{1}} \wedge d z_{h_{2}} \wedge \cdots \wedge d z_{h_{k}} .
$$

(2) suppose that $h_{1} \neq 1$ : from equation (6) we have

$$
g_{h_{1} \ldots h_{k}}\left(z_{1}, \ldots, z_{n}\right)=c_{\tilde{m}}^{h_{1} \ldots h_{k}} z_{1}^{m_{1}} z_{j_{2}}^{m_{j_{2}}} \ldots z_{j_{n-k}}^{m_{j_{n-k}}}
$$

where $\left\{j_{1}=1, j_{2}, \ldots, j_{n-k}\right\}=\{1, \ldots, n\} \backslash\left\{h_{1}, \ldots, h_{k}\right\}$, and $m_{h_{1}}=\cdots=$ $m_{h_{k}}=1$.

Since $g_{h_{1} \cdots h_{k}}$ is not zero along $V_{1}$, we must have $m_{j_{2}}=\cdots m_{j_{n-k}}=0$. Now we have two subcases:

1a) If $m_{1}=0$, we are in the previous case $m_{h_{1}}=\cdots=m_{h_{k}}=1$ and $m_{j}=0$ in the other cases, and therefore

$$
\pi^{*} \omega=C d z_{h_{1}} \wedge d z_{h_{2}} \wedge \cdots \wedge d z_{h_{k}}
$$

1b) If $m_{1}=1$, we have $m_{h_{1}}=\cdots=m_{h_{k}}=1$ and $m_{j_{2}}=\cdots m_{j_{n-k}}=0$ in the other cases. Again, as now we know all $m_{i}^{\prime} s$, from equation 6, we have that the only coefficients of the $k$-form $\pi^{*} \omega$ which is not a null monomial is

$$
g_{h_{1} \ldots h_{k}}=C z_{1}, g_{1 h_{2} \ldots h_{k}}=C_{1} z_{h_{1}}, g_{1 h_{1} h_{3} \cdots h_{k}}=C_{2} z_{h_{2}}, \cdots, g_{1 h_{1} h_{2} \cdots h_{k-1}}=C_{k} z_{h_{k}}
$$

Since $k+1 \leq n-1$, the $k$-form $\pi^{*} \omega$ which induces the Pfaff system is not regular. A contradiction. 
Therefore any regular holomorphic Pfaff system on a no-resonance Hopf manifold $X=W /\langle f\rangle$ is induced by a constant $k$-form of the type

$$
\pi^{*} \omega=C d z_{i_{1}} \wedge \cdots \wedge d z_{i_{k}}, C \in \mathbb{C}^{*} .
$$

That is, it is always integrable and has a compact leaf $W_{k} /\langle f\rangle$, where

$$
W_{k}=\left\{z_{i_{1}}=\cdots=z_{i_{k}}=0\right\}-\{0\} .
$$

Weak no-resonance case. In this case $\mu_{1}=\cdots=\mu_{r}$, then by Lemma 3.2 item (iii) we have

Then from (4) we get

$$
b=\mu^{m} \mu_{r+1}^{m_{r+1}} \ldots \mu_{n}^{m_{n}}
$$

$$
\mu^{\alpha_{1}+\cdots+\alpha_{r}} \mu_{r+1}^{\alpha_{r+1}} \ldots \mu_{n}^{\alpha_{n}}=\mu_{i_{1}}^{-1} \ldots \mu_{i_{k}}^{-1} \mu^{m} \mu_{r+1}^{m_{r+1}} \ldots \mu_{n}^{m_{n}},
$$

for all $\alpha=\left(\alpha_{1}, \alpha_{2}, \ldots, \alpha_{n}\right) \in \mathbb{N}^{n}$ and $i_{1}<\cdots<i_{k}$ such that $c_{\alpha}^{i_{1} \ldots i_{k}} \neq 0$. Since there is not non-trivial relation between $\mu, \mu_{r+1}, \ldots, \mu_{n}$ 's we have:

for $s \in\{0, \ldots, k\}$ such that $i_{1}, \ldots, i_{s} \leq r$ and $r+1 \leq i_{s+1}, \ldots, i_{k}$, then

$$
\alpha_{1}+\cdots+\alpha_{r}=m-s
$$

and $\left(\alpha_{r+1}, \ldots, \alpha_{n}\right)=\left(m_{r+1}, \ldots, m_{i_{s+1}}-1, \ldots, m_{i_{k}}-1, \ldots, m_{n}\right)$. Therefore

$$
g_{i_{1} \ldots i_{k}}=z_{r+1}^{m_{r+1}} \ldots z_{i_{s+1}}^{m_{i_{s+1}}-1} \ldots z_{i_{k}}^{m_{i_{k}}-1} \ldots z_{n}^{m_{n}} \cdot \sum_{\alpha_{1}+\ldots+\alpha_{r}=m-s} c_{\alpha}^{i_{1} \ldots i_{k}} z_{1}^{\alpha_{1}} \ldots z_{r}^{\alpha_{r}}
$$

By the same reason in the no-resonance case, we must have that $m_{i} \in\{0,1\}$, for all $i \geq r+1$. Then if the coefficient $g_{i_{1} \ldots i_{k}} \not \equiv 0$, we have from (7) that

$$
g_{i_{1} \ldots i_{k}}=z_{j_{1}}^{m_{j_{1}}} \ldots z_{j_{n-r-k+s}}^{m_{j_{n-k+s}}} \cdot \sum_{\alpha_{1}+\cdots+\alpha_{r}=m-s} c_{\alpha}^{i_{1} \ldots i_{k}} z_{1}^{\alpha_{1}} \ldots z_{r}^{\alpha_{r}}
$$

where $\left\{j_{1}, \ldots, j_{n-r-k+s}\right\}=\{r+1, \ldots, n\} \backslash\left\{i_{s+1}, \ldots, i_{k}\right\}$ and $m_{i_{s+1}}=\cdots=m_{i_{k}}=$ 1.

\subsection{Examples.}

Example 3.3. Let $X=\left(\mathbb{C}^{5}-\{0\}\right) /<\mu>$ be a classical Hopf manifold. The 2-form

$$
\omega=z_{3} d z_{1} \wedge d z_{2}+z_{1} d z_{1} \wedge d z_{3}+z_{2} d z_{1} \wedge d z_{4}+z_{5} d z_{1} \wedge d z_{5}+z_{4} d z_{4} \wedge d z_{5}
$$

induces a regular codimension two Pfaff system on $X$.

\subsection{Examples.}

Example 3.4. Let $X=\left(\mathbb{C}^{5}-\{0\}\right) /<\mu_{1} \cdots \mu_{5}>$ be a no-resonance Hopf manifold. The 3 -form

$$
\omega=z_{4} z_{5} d z_{1} \wedge d z_{2} \wedge d z_{3}+z_{1} z_{3} d z_{2} \wedge d z_{4} \wedge d z_{5}
$$

induces a singular codimension three Pfaff system on $X$.

Example 3.5. Let $X=\left(\mathbb{C}^{6}-\{0\}\right) /<\mu \mu \mu \mu_{4} \mu_{5} \mu_{6}>$ be a weak no-resonance Hopf manifold. The 2-form

$$
\omega=z_{5} z_{6} d z_{1} \wedge d z_{4}+z_{2} z_{4} d z_{5} \wedge d z_{6}
$$

induces a singular codimension two Pfaff system on $X$. 


\section{Proof of Theorem 1.5}

Let $X$ be a no-resonance Hopf manifold of dimension $n$.

Definition 4.1. Let $\mathcal{F}$ be a distribution on a Hopf manifold $X=\mathbb{C}^{n}-\{0\} /\langle f\rangle$ with tangent sheaf $T \mathcal{F} \subset T X$, and $G \subset G L(n, \mathbb{C})$ a subgroup. We say that $\mathcal{F}$ is $G$-invariant distribution if the pullback of $T \mathcal{F}$ to $\mathbb{C}^{n}$ is invariant with respect to the natural $G$-action on $\mathbb{C}^{n}$.

Proposition 4.2. Let $G=\left(\mathbb{C}^{*}\right)^{n} \subset G L(n, \mathbb{C})$ be the diagonal subgroup, and $\mathcal{F} \subset$ $T \mathbb{C}^{n}$ a $G$-invariant distribution. Then $\mathcal{F}$ is integrable.

Proof. Since the Frobenius form, representing the obstruction to integrability, is continuous, it would suffice to prove 4.2 is general point of $\mathbb{C}^{n}$. Therefore, it would suffice to prove it on an open orbit of $G$, which is identified with $G$. A left-invariant distribution $B \subset T G$ is integrable. Indeed, $B$ is generated by left-invariant vector fields, which commute, because $G$ is commutative.

The integrability of the distribution will follow from Proposition 4.2 and the following theorem.

Theorem 4.3. Let $\mathcal{F}$ be a holomorphic distribution on a no-resonance Hopf manifold $X=\mathbb{C}^{n}-\{0\} /\langle f\rangle$. Denote by $G$ the group $\left(\mathbb{C}^{*}\right)^{n}$ of diagonal matrices commuting with $f$. Then $\mathcal{F}$ is $G$-invariant.

Proof. Step 1: Let $G_{f}$ be the Zariski closure of the group $\langle f\rangle \cong \mathbb{Z}$. This is an algebraic group, which is obtained as the smallest algebraic group containing $\langle f\rangle$. This implies that $G_{f} \subset G$. However, any algebraic subgroup of $G$ is given by a set of equations of form $\prod_{i} z_{i}^{n_{i}}=1$, hence none of them contains $\langle f\rangle$. We have shown that $G_{f}=G$.

Step 2: Distributions on a manifold $X$ can be interpreted as subvarieties in the total space $T X$ of a tangent bundle, which are closed under the natural algebraic operations (addition and multiplication by a number).

Let $J \subset \mathcal{O}_{T X}$ be the ideal corresponding to $\mathcal{F}$. Denote by $\tilde{J} \subset \mathcal{O}_{\mathbb{C}^{2 n}}$ the ideal $j_{*} \pi^{*}(J)$, where $\pi: \mathbb{C}^{n}-\{0\} \rightarrow X$ is the quotient map and $j: \mathbb{C}^{n}-\{0\} \rightarrow \mathbb{C}^{n}$ the standard embedding. Then $\tilde{J}$ is a $f$-invariant ideal sheaf in $\mathcal{O}_{\mathbb{C}^{2 n}}$.

To finish the proof it remains to show that any $\langle f\rangle$-invariant ideal in $\mathcal{O}_{T M}$ is invariant with respect to the algebraic closure of $\langle f\rangle$. This is implied by the following lemma, which finishes the proof.

Lemma 4.4. Let $f: \mathbb{C}^{n} \rightarrow \mathbb{C}^{n}$ be a linear, invertible holomorphic contraction, and $I \subset \mathcal{O}_{\mathbb{C}^{2 n}}$ a $f$-invariant ideal. Then $I$ is $G$-invariant, where $G$ is Zariski closure of $f$ in $G L(n, \mathbb{C})$.

Proof. Let us call a holomorphic function on $\mathbb{C}^{n} f$-finite if it is contained in finitedimensional, $f$-invariant subspace in $H^{0}\left(\mathbb{C}^{2 n}, \mathcal{O}_{\mathbb{C}^{2 n}}\right)$. As follows from OV2, Theorem 4.2], $f$-finite functions are dense in the space of all holomorphic functions on $\mathbb{C}^{n}$. 
The same argument also proves that the $f$-finite sections of $I$ are dense in the space of all sections of $I$. However, by definition of Zariski closure, any finitedimensional $f$-invariant subspace is also $G$-invariant, hence $I$ is also $G$-invariant.

\section{REFERENCES}

[CFF] M. Corrêa, A. Fernández-Pérez, A. M. Ferreira: Classification of holomorphic foliations on Hopf manifolds. Mathematische Annalen. v. 365, (1) , 579-593, 2016.

[CJV] M. Corrêa Jr, M. Jardim, R. Vidal, On the Singular scheme of split foliations, Indiana University Mathematics Journal, 64 No. 5 1359-1381, 2015.

[CMS] M. Corrêa Jr , L. G. Maza, M. G. Soares, Hypersurfaces Invariant by Pfaff Equations, Communications in Contemporary Mathematics, 17, 1450051. 2015.

[Da] K. Dabrowski, Moduli Spaces for Hopf Surfaces. Math. Ann. 259 (1982) 201-225.

[EK] E. Esteves and S. Kleiman, Bounding solutions of Pfaff equations. Comm. Algebra 31 (2003), 3771-3793.

[G] E. Ghys, Feuilletages holomorphes de codimension un sur les espaces homogenes complexes. Ann. Fac. Sci. Toulouse Math. (6) 5 (1996), no. 3, 493-519.

[Ha] A. Haefliger: Deformations of transversely holomorphic flows on spheres and deformations of Hopf manifolds.Compositio Mathematica,55 (1985), 241-251.

[Ho] H. Hopf, Zur Topologie der komplexen Mannigfaltigkeiten, Courant Birthday Volume, pp. 167-185, New York 1948.

[I] M. Ise, On the geometry of Hopf manifolds. Osaka Math. J. 12 (1960), 387-402.

[Kat] Ma. Kato, Some Remarks on Subvarieties of Hopf Manifolds, Tokyo J. Math. 2, Nr. 1 (1979), 47-61.

[Ko] K. Kodaira, On the structure of compact complex analytic surfaces II, Amer. J. Math. 88 (1966) 682-721.

[Ma1] D. Mall, On holomorphic and transversely homomorphic foliations on Hopf surfaces. J. reine angew. Math, 501 (1998), 41-69.

[Ma2] D. Mall, The cohomology of line bundles on hopf manifolds. Osaka J Math. 28, (1991), 999-1015.

[Me] A. de Medeiros, Singular foliations and differential p-forms. Ann. Fac. Sci. Toulouse Math. (6) 9 (2000), no. 3, 451-466.

[OV1] L. Ornea, M. Verbitsky, Locally conformal Kähler manifolds with potential, Math. Ann. 348 (2010), 25-33.

[OV2] L. Ornea, M. Verbitsky, Embedding of LCK manifolds with potential into Hopf manifolds using Riesz-Schauder theorem, Complex and Symplectic Geometry, Springer INdAM serie, 2017, 137-148.

[S] S. Sternberg, Local contractions and a theorem of Poincaré, Amer. J. Math. 79 (1957), 809-824.

[W] J. Wehler, Versal deformation of Hopf surfaces. J. reine angew. Math.328, 1981, 22-32.

Maurício Corrêa, ICEx - Ufmg, Departamento de Matemática, Av. Antônio Carlos 6627, 30123-970 Belo Horizonte MG, Brazil

Email address: mauriciojr@ufmg.br

Antonio M. Ferreira, DeX - Ufla, Av P.H. Rolfs, s/n, Campus Universitário, Lavras MG, BRAZIL, CEP 37200-000

Email address: antoniosilva@dex.ufla.br

Misha Verbitsky, impa-Instituto Nacional de Matemática Pura e Aplicada, Estrada Dona Castorina, 110, Jardim Botânico, CEP 22460-320, Rio de Janeiro, RJ -Brazil, and, Laboratory of Algebraic Geometry, National Research University Higher School of Economics, 6 Usacheva Str., Moscow, Russia., Misha Verbitsky is supported by Russian Academic Excellence Project '5-100", CNPQ - Process 313608/2017-2, And FAPERJ E26/202.912/2018.

Email address: verbit@impa.br 